\section{Testing the Strong-Stretching Assumption in a Block Copolymer Microstructure}

\section{W. Matsen* and F. S. Bates}

Department of Chemical Engineering and Materials Science, University of Minnesota, Minneapolis, Minnesota 55455

Received August 29, 1995

Strong-segregation theory (SST) ${ }^{1}$ models microphaseseparated block copolymer melts in terms of polymeric brushes. This approach has provided intuitive and wellaccepted explanations for phase behavior far below the order-disorder transition. According to the model, $A B$ diblock copolymers self-assemble with their junction points along narrow interfaces. The $A$ and $B$ blocks form brushes on opposite sides of the interface. Due to interfacial tension, copolymers pack tightly, producing strongly stretched brushes. For symmetric diblocks, the interfaces remain flat and the brushes stack together to form a lamellar phase, as depicted in Figure 1. For asymmetric diblocks, the interfaces curve due to the unbalanced elastic energies of the $A$ and $B$ brushes, forming, for example, cylinders or spheres with the shorter blocks on the inside. These units then pack together to form an ordered microstructure. Accepting this model, it is possible to describe diblock melts with the analytical theory developed for brushes. ${ }^{2,3}$

The SST ignores interpenetration between adjacent brushes, which is valid only if the brushes are strongly stretched. Although the strong-stretching assumption has been supported by theoretical arguments, ${ }^{2,4}$ there exists evidence to the contrary. ${ }^{5}$ Considering the extensive use of SST, $, 1,3,6$ this issue demands further attention. In addition to concerns regarding the validity of SST, interpenetration is important in regard to a number of phenomena, such as how shear causes microstructures to orient ${ }^{7}$ and how microstructures wet solid surfaces. ${ }^{8}$ Self-consistent field theory (SCFT) $)^{9,10}$ is well suited for examining the true extent of this interpenetration at the various degrees of block incompatibility. It accounts for all copolymer configurations and weights them by the appropriate Boltzmann factor. In this way, finite-segregation effects, not normally included in the SST, are properly treated.

In this Communication, we present a SCFT calculation examining the penetration between $\mathrm{AB}$ diblocks from adjacent interfaces of the lamellar microstructure. This calculation is restricted to the symmetric case where both blocks have polymerization indexes of $N / 2$ (i.e., $f=1 / 2$ ) and both segments have equal statistical lengths $\left(\alpha_{\mathrm{A}}=a_{\mathrm{B}} \equiv a\right)$; the theory is easily generalized to the asymmetric case, $f \neq 1 / 2$ or $a_{\mathrm{A}} \neq a_{\mathrm{B}}$. Immiscibility between $\mathrm{A}$ and $\mathrm{B}$ segments is controlled through the Flory-Huggins parameter, $\chi$. Contrary to the SST arguments, ${ }^{2,4}$ we find that the degree of penetration is substantial even up to very high degrees of segregation $\left(\chi N \sim 10^{3}\right)$. We propose that it is primarily due to the entropy associated with the distribution of end segments. Incorporating this free energy contribution into SST produces behavior consistent with our SCFT calculation.

The SCFT for an $A B$ diblock melt is described in ref 11. However, this reference does not describe how to calculate the end-segment distribution for polymers from just a single interface. We do that now. As in ref 11 , each diblock is parameterized with $t^{\prime}=0-1 / 2$ along the $\mathrm{A}$ block and $t^{\prime}=1 / 2-1$ along the $\mathrm{B}$ block. For the

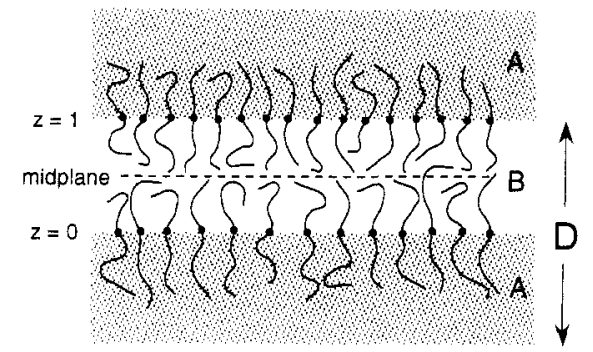

Figure 1. Strongly segregated diblock copolymers forming a lamellar microstructure.

symmetric diblocks considered here, all the lamellae are equivalent, and so only a B-rich layer needs to be considered. Following ref 11 , we first evaluate $D$, the equilibrium lamellar spacing, and $\omega_{\mathrm{B}}(\mathbf{r})$, the field acting on $B$ segments at position $\mathbf{r}$. This calculation also provides $q(\mathbf{r}, t)$, and $q^{\dagger}(\mathbf{r}, t)$, the partition functions for the $t^{\prime}<t$ and $t^{\prime}>t$ portions, respecitvely, of a chain with segment $t$ constrained at $\mathbf{r}$. With these quantities, we can determine $P(\mathbf{r}, t)$, the probability distribution of segment $t$ from those copolymers with their junctions (i.e., segment $t^{\prime}=1 / 2$ ) confined to a particular interface. Noting that the distribution of copolymer junctions in the melt is proportional to $q(\mathbf{r}, 1 / 2) q^{\dagger}(\mathbf{r}, 1 / 2)$, the distribution of those at a single interface, $P(\mathbf{r}, 1 / 2)$, is evaluated. Then this distribution is propagated along the B block from $t=1 / 2$ to 1 using

$$
P(\mathbf{r}, t)=\int \mathrm{d} \mathbf{r}^{\prime} P\left(\mathbf{r}^{\prime}, t^{\prime}\right) G\left(\mathbf{r}, t ; \mathbf{r}^{\prime}, t^{\prime}\right)
$$

where $G\left(\mathbf{r}, t: \mathbf{r}^{\prime}, t^{\prime}\right)$ is the probability of finding segment $t$ at $\mathbf{r}$ given that segment $t^{\prime}$ is at $\mathbf{r}^{\prime}$. Provided that $\epsilon \equiv t$ $-t^{\prime}>0$ is small,

$$
\begin{aligned}
G\left(\mathbf{r}, t ; \mathbf{r}^{\prime}, t^{\prime}\right)= & \left(\frac{3}{2 \pi N a^{2} \epsilon}\right)^{3 / 2} \frac{q^{\dagger}(\mathbf{r}, t)}{q^{\dagger}\left(\mathbf{r}^{\prime}, t^{\prime}\right)} \\
& \exp \left(-\frac{3}{2 N a^{2} \epsilon}\left|\mathbf{r}^{\prime}-\mathbf{r}\right|^{2}-\epsilon \omega_{B}(\mathbf{r})\right)
\end{aligned}
$$

Reference 9 explains how to convert eqs 1 and 2 into a differential equation. For a lamellar phase,

$$
\frac{\partial p}{\partial t}=\frac{2}{3} \Delta^{-2} \frac{\partial^{2} p}{\partial z^{2}}-\omega_{B}(z) p
$$

where $p(z, t) \equiv P(z, t) / q^{\dagger}(z, t), \Delta \equiv D / a N^{1 / 2}$, and $z$ is a dimensionless coordinate varying from 0 to 1 across the $\mathrm{B}$-rich lamella. The $z=0$ interface is defined to be the one from which the B blocks originate, and the distribution of their free ends is $g(z) \equiv P(z, 1)$. In terms of $g(z)$, the fraction of blocks with ends extending beyond the $z$ $=1 / 2$ midplane, $\nu$, and the dimensionless penetration depth, $\xi$, are defined by

$$
\begin{gathered}
v=\int_{1 / 2}^{\infty} \mathrm{d} z g(z) \\
g\left(\frac{1}{2}+\frac{1}{2} \xi\right)=\frac{1}{2} g\left(\frac{1}{2}\right)
\end{gathered}
$$

We now describe an analogous calculation using the SST. To begin, consider a B block extending from $z=$ 0 to $z=y$. The length, $l$, of the segment at $z=x(0 \leq$ $x \leq y)$ defines the function $E(x, y) \equiv 2 N l / D$. In terms of this function, the free energy per molecule in units of $k_{\mathrm{B}} T$ is $f \equiv F / n k_{\mathrm{B}} T=e+\gamma-s$ where 


$$
\begin{gathered}
e=\frac{3}{4} \Delta^{2} \int_{0}^{1} \mathrm{~d} y \int_{0}^{y} \mathrm{~d} x g(y) E(x, y) \\
\gamma=2 \Delta^{-1} \sqrt{\chi N / 6} \\
s=-2 \int_{0}^{1} \mathrm{~d} y g(y) \ln (g(y) / \Delta)
\end{gathered}
$$

The first contribution, $e$, is the elastic energy of stretching the copolymers. The second, $\gamma$, is the interfacial tension between the $\mathrm{A}$ and $\mathrm{B}$ domains. The last term is the entropy associated with the distribution of end segments. (The factor of $\Delta$ inside the logarithm is necessary because we define $g(y)$ to be dimensionless.) To obtain $g(y)$, we minimize $f$ with respect to $g(y), E(x, y)$, and $\Delta$ subject to the constraints that the melt is incompressible and each block has a degree of polymerization N/2. Mathematically, the two constraints are

$$
\begin{gathered}
\int_{x}^{1} \frac{\mathrm{d} y g(y)}{E(x, y)}+\int_{1-x}^{1} \frac{\mathrm{d} y g(y)}{E(1-x, y)}=1 \\
\int_{0}^{y} \frac{\mathrm{d} x}{E(x, y)}=\frac{1}{2}
\end{gathered}
$$

(The combination of these two constraints ensures that $\int \mathrm{d} y g(y)=1$.) The minimization is performed numerically following the procedure in ref 12 .

The new aspect of the present SST calculation is the treatment of the entropy term, eq 8 . It is derived by considering $A(y)$, the partition function for a $\mathrm{B}$ block stretched between $z=0$ and $z=y$. In terms of $A(y)$, the average energy of the $\mathrm{B}$ block in units of $k_{\mathrm{B}} T$ is $-f \mathrm{~d} y$ $g(y) \ln A(y)$, and the free energy is $-\ln Z$, where $Z=$ $D \int \mathrm{d} y A(y)$. The difference between the energy and free energy is the entropy. Noting that $g(y)=D A(y) / Z$ and that each copolymer has two blocks leads immediately to eq 8 . In the $\chi N \rightarrow \infty$ limit, the entropy, $s$, is irrelevant and the minimization can be performed analytically. ${ }^{1}$ This produces the solution $g(z)=4 z /\left(1-4 z^{2}\right)^{1 / 2}$, for $0<$ $z<1 / 2$ and $g(z)=0$ otherwise, which implies that $\nu=\xi$ $=0$. In this limit, the stretching energy is $e=\pi^{2} \Delta^{2} / 32$, which produces a lamellar spacing of $\Delta=2\left(8 \times N / 3 \pi^{4}\right)^{1 / 6}$. Including the entropy, $s$, drives $g(z)$ toward a more uniform distribution, causing $e$ to increase by typically 0.5 . At finite, realistic values of $\chi N$, this is too significant to ignore.

Our results for $g(z)$ are plotted in Figure 2. Those for the SCFT are shown at $\chi N=20,100$, and 1000. Even the most segregated example shows considerable penetration beyond the midplane and is poorly approximated by the infinite $\chi N$ limit. However, when the entropy term in eq 8 is accounted for, the SST produces good agreement at $\chi N=1000$. The SST becomes less accurate as the segregation decreases, but is still reasonable for $\chi N=100$. In the SCFT, $g(z)$ can extend beyond the range $0 \leq z \leq 1$ because $B$ blocks are not strictly confined to B-rich lamellae, whereas in the SST, $g(z)$ is strictly 0 outside this interval. Nevertheless, $g(0)$ and $g(1)$ are both nonzero in the SST with a particularly large discontinuity at $z=0$. Interestingly, the SCFT produces a shoulder near $z=0$ consistent with that discontinuity.

Figure 3 shows the fraction of ends extending beyond the midplane $v$ and the dimensionless penetration depth $\xi$ as a function of $\chi N$. For $\chi N>100$, the SST results corrected for the entropy of the end segments agree well with the SCFT ones. Recall that without the correction,

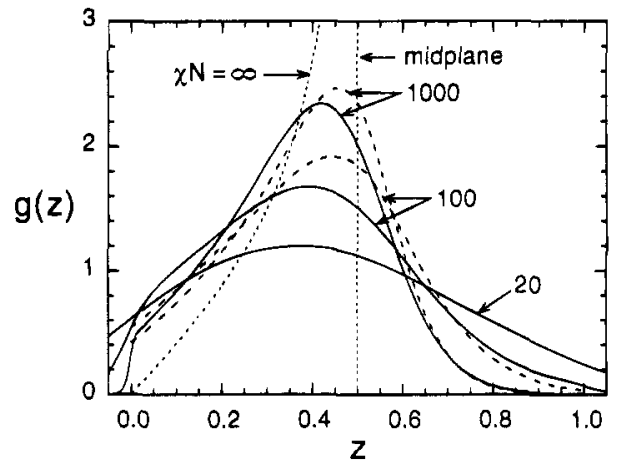

Figure 2. End-segment distribution for blocks originating from an interface at $z=0$. Curves obtained from the SCFT and SST are shown with solid and dashed lines, respectively. The infinite $\chi N$ limit is denoted with a dotted line.

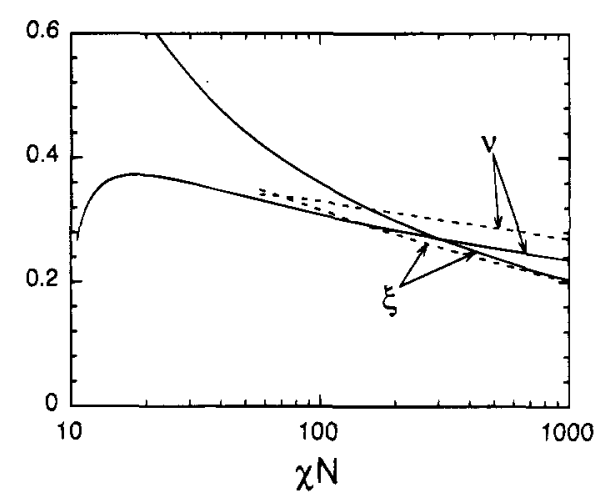

Figure 3. Fraction of ends extending beyond the midplane, $v$, and the penetration depth, $\xi$, as a function of segregation. Solid curves are obtained with SCFT, and dashed ones with SST.

SST predicts $v=\xi=0$. Clearly, these results demonstrate that the strong-stretching limit examined in ref 4 , where $v, \xi \ll 1$, is not relevant to diblock microstructures.

Although it becomes computationally prohibitive to extend our SCFT calculations beyound $\chi N \sim 10^{3}$, the SST results can be pushed to ultrahigh degrees of segregation, $\chi N \sim 10^{5}$. At segregations of this magnitude, our SST results do agree with those in refs 2 and 4. For instance, $\xi \approx 1.11 \Delta^{-4 / 3}$, consistent with their predicted exponent. Furthermore, the tail of the endsegment distribution fits the functional form $g(z) \approx g_{0}$ $\exp \left(-1.9((z-1 / 2) / \xi)^{3 / 2}\right)$ well for $z-1 / 2 \gg \xi$. The proportional factor $g_{0}$ is of order unity and depends weakly on $\xi$.

The present calculations illustrate that the brush description of block copolymer microphases is inaccurate for what is experimentally considered strong segregations (i.e., $\chi N \sim 100$ ). For such segregations, the unfavorable enthalpic interactions do cause nearly complete segregation of the $\mathrm{A}$ and $\mathrm{B}$ blocks with relatively narrow interfaces, ${ }^{10}$ as assumed in the SST. However, the interfacial tension is not sufficient to produce strongly stretched brushes. The implication is that SST cannot be relied upon to quantitatively predict experimental results. Despite this shortcoming, SST still provides qualitative predictions and intuitive explanations. Furthermore, it provides a foundation to which entropy corrections can be applied, like the one presented here. Our results should not be considered a reason to dismiss the SST, but rather a word of caution regarding its accuracy and motivation for future improvements. 
Acknowledgment. This work was supported by the NSF (Grant DMR 94-05101) and by the Minnesota Supercomputer Institute.

\section{References and Notes}

(1) Semenov, A. N. Sov. Phys. JETP 1985, 61, 733.

(2) Milner, S. T. Science 1991, 251, 905.

(3) Milner, S. T. J. Polym. Sci., Part B 1994, 32, 2743.

(4) Witten, T. A.; Leibler, L.; Pincus, P. A. Macromolecules 1990, 23,824 .

(5) Shull, K. R.; Mayes, A. M.; Russell, T. P. Macromolecules 1993, 26, 3929 .
(6) Olmsted, P. D.; Milner, S. T. Phys. Rev. Lett. 1994, 72, 936; 1995, 74, 829. Likhtman, A. E.; Semenov, A. N. Macromolecules 1994, 27, 3103 .

(7) Koppi, K. A.; Tirrel, M.; Bates, F. S.; Almdal, K.; Colby, R. H. J. Phys. II Fr. 1992, 2, 1941.

(8) Sikka, M.; Singh, N.; Bates, F. S.; Karim, A.; Satija, S.; Majkrzak, C. F. J. Phys. II Fr. 1994, 4, 2231.

(9) Helfand, E. J. Chem. Phys. 1975, 62, 999.

(10) Matsen, M. W.; Bates, F. S. Macromolecules, in press.

(11) Matsen, M. W.; Schick, M. Phys. Rev. Lett. 1994, 72, 2660.

(12) Matsen, M. W. J. Chem. Phys. 1995, 102, 3884.

MA951279K 